\title{
Optimal number and position of the magnetic sensors for plasma shape identification in ITER ${ }^{\star}$
}

\author{
Marco Ariola ${ }^{1}$ \\ Consorzio CREATE, Dipartimento di Ingegneria, Università degli Studi di Napoli Parthenope \\ Centro Direzionale di Napoli, Isola C4, 80143 Napoli, Italy \\ E-mail: ariola@uniparthenope.it
}

\author{
Alfredo Pironti, Raffaele Albanese, Giuseppe Ambrosino \\ Consorzio CREATE, Dipartimento di Ingegneria Elettrica e Tecnologie dell'Informazione, Università \\ degli Studi di Napoli Federico II \\ Via Claudio 21, 80125 Napoli, Italy \\ E-mail: $\{$ pironti, ralban, ambrosin\} eunina.it
}

\section{Shakeib Arshad \\ Fusion for Energy \\ 08019 Barcelona, Spain}

E-mail: Shakeib.Arshadef4e.europa.eu

\section{George Vayakis}

ITER Organization

Route de Vinon sur Verdon, 13115 Saint Paul-lez-Durance, France

E-mail: george.vayakiseiter.org

\begin{abstract}
Position and number of the magnetic sensors affect the accuracy of the plasma shape identification algorithms. These algorithms usually solve an inverse problem based on the solution of the Grad-Shafranov equilibrium equation, and they need as input a certain number of magnetic field and flux measurements in points located around the plasma. The scope of this paper is to investigate on the problem of assessing the minimum number of magnetic sensors that are necessary for the solution of the plasma shape identification in a tokamak machine. In particular, with reference to the ITER inner vessel magnetic diagnostic system, it is shown that, adopting a heuristic procedure, the available measurements are divided in two subsets, where the measurements in the first subset can be reconstructed by means of suitable linear combinations of measurements in the second subset. In this way, it is possible to evaluate the level of the redundancy of the ITER inner vessel diagnostic system and to outline a suitable fault management system.
\end{abstract}

First EPS Conference on Plasma Diagnostics - $1^{\text {st }}$ ECPD

14-17 April 2015,

Villa Mondragone, Frascati (Rome) Italy

\footnotetext{
*The work reported was funded partially by Fusion for Energy under Grant F4E-GRT-047. This publication reflects the views of the authors only. Fusion for Energy cannot be held responsible for any use which may be made of the information contained herein. The work has also been partially funded by the Italian MIUR under PRIN grant 2010SPS9B3.

${ }^{1}$ Speaker
} 


\section{Introduction}

Plasma confinement is the main problem for civil application of nuclear fusion; currently tokamak devices are the most common solution to this problem. Tokamaks, first built in the former Soviet Union at the end of 1960's, are machines characterized by toroidal geometry, where the confinement is obtained via the interaction of the plasma with an external electromagnetic field. Unfortunately, when high elongation is required, the equilibrium configuration between the plasma column and the external field is unstable, i.e. small perturbations from the equilibrium may cause large movements of the plasma. Hence the use of a feedback system for current, position and shape control becomes mandatory [2].

Measurement of some significant quantities such as the plasma-wall gaps, the plasma current, the poloidal beta, etc., is essential for magnetic control of tokamak plasmas. Since most of the relevant parameters are not directly measurable, they must be estimated starting from the set of available data [3]. The scope of this paper is to develop a method that enables determination of the minimum number of sensors that are needed to reconstruct the quantities which are usually used for plasma magnetic control. A general solution to this problem is beyond the scope of this paper; we will concentrate our analysis on the ITER tokamak (see [5]), which represents the next major step toward harnessing nuclear fusion.

The paper is structured as follows: in Section II we describe the magnetic sensors that are going to be placed on ITER for equilibrium reconstruction; Section III describes the optimization problems that have been tackled and the way they have been solved; Section IV presents the simulation results obtained adopting the proposed procedure making use of the reconstruction code EFIT++ [1]; finally Section V presents conclusions of this work.

\section{The ITER magnetic diagnostics and the virtual experiment database}

The magnetic sensors that are envisaged to be placed in the ITER machine for the purpose of equilibrium reconstruction consist of 78 primary sensors, inside the tokamak inner vessel, shown in Fig.1, divided into: 24 tangential pickup coils; 12 normal pickup coils; 12 pickup coils in the divertor region; 22 saddle coils; 8 full flux loops.
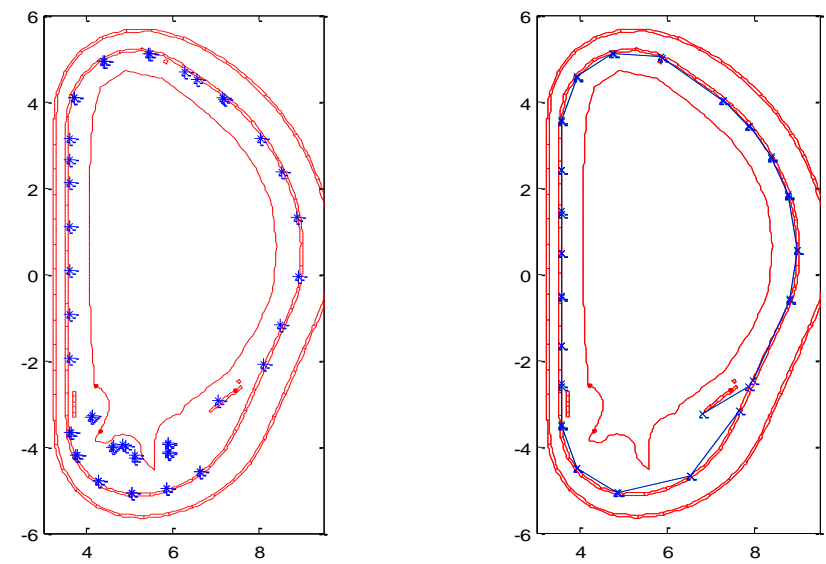

Fig. 1. The ITER magnetic pickup coils on the left, and saddle and full flux loops on the right. 
In our analysis, we assume that each sensor is affected by measurement noise; for the generic sensor $i$ the noisy measurement is modeled as

$$
y_{n_{i}}=\left(1+n_{r_{i}}\right) y_{i}+n_{a_{i}}
$$

where the multiplicative and additive noise signals are assumed to be zero mean gaussian independent processes with assigned standard deviations $\sigma_{r_{i}}$ and $\sigma_{a_{i}}$, respectively.

Samples of the exact measurements have been obtained by considering a database of $N=552$ plasma equilibria. The equilibria in the database are representative of the ITER plasma scenarios, with plasma flat-top currents ranging from 2.25MA to 17MA. Considering each one of these plasma equilibrium configurations as a virtual experiment, it is reasonable to assume that the virtual experiments adequately cover ITER operating space for the purposes of this study.

Because of the presence of the multiplicative term in (1), the standard deviation of the measurement error due to noise depends on the generic virtual experiment $l(l=1,2, \ldots, N)$

$$
\sigma_{i}(l)=\sqrt{E\left[y_{n_{i}}(l)-y_{i}(l)\right]^{2}}=\sqrt{\sigma_{r_{i}}^{2} y_{i}^{2}(l)+\sigma_{a_{i}}^{2}}
$$

where $E$ denotes the expected value operator.

We introduce two bounds for the standard deviation estimation error by considering the minimum and the maximum value of $y_{i}^{2}(l)$ over the virtual experiments database, obtaining $\sigma_{i}^{\prime} \leq \sigma_{i}(l) \leq \sigma_{i}^{\prime \prime}$, where $\sigma_{i}^{\prime}=\min _{l=1,2, \ldots, N} \sigma_{i}(l)$ and $\sigma_{i}^{\prime \prime}=\max _{l=1,2, \ldots, N} \sigma_{i}(l)$.

With this in mind, in what follows we will consider an additive model for the measurement noise, in the form

$$
y_{n_{i}}=y_{i}+n_{i}
$$

where $n_{i}$ is a zero mean gaussian process. Depending on the type of analysis we will consider as standard deviation of $n_{i}$ the value $\sigma_{i}^{\prime}$ or the value $\sigma_{i}^{\prime \prime}$.

\section{Problem formulation and solution}

The scope of this section is to tackle the problem of choosing a subset of sensors whose measurements are able to reconstruct the whole set, with a given level of accuracy.

Let us introduce a matrix $\mathrm{F} \in \mathbb{R}^{\mathrm{M} \times \mathrm{N}}$

$$
F=\left[\begin{array}{llll}
f_{1} & f_{2} & \ldots & f_{M}
\end{array}\right]^{T},
$$

where the $k$-th row is the vector containing the measurement of the sensor $k$ for all the $N$ virtual experiments. We will assume that the number of experiments is much greater than the number of sensors, i.e. $M \ll N$.

Let us now consider the following problem. 
Problem 1. Find a partition of the sensor set indices $\{1,2, \ldots, M\}$, into two sets $\left\{j_{1}, j_{2}, \ldots, j_{p}\right\}$ and $\left\{h_{1}, h_{2}, \ldots, h_{m}\right\}$, and $p$ vectors $k_{1}, k_{2}, \ldots, k_{p}$, of dimension $m$, such that the $p$ measurements of the first set can be obtained as linear combinations of the $m$ measurements of the second set, with coefficient vector $k_{i}$, with a prescribed accuracy in the presence of noise.

Let $\left\{h_{1}, h_{2}, \ldots, h_{m}\right\} \subset\{1,2, \ldots, M\}$, and consider the sensor number $j \notin\left\{h_{1}, h_{2}, \ldots, h_{m}\right\}$. Let us denote by $\tilde{f}_{j}$ an approximation of the vector $f_{j}$ obtained by a linear combination of the measurements of the sensor $h_{1}, h_{2}, \ldots, h_{m}$, through the vector $k_{j} \in \mathbb{R}^{m}$. The generic element of $\tilde{f}_{j}$, which depends on the virtual experiment $l$, is given by

$$
\tilde{f}_{j}(l)=k_{j}^{T}\left[\begin{array}{c}
f_{h_{1}}(l) \\
f_{h_{2}}(l) \\
\vdots \\
f_{h_{m}}(l)
\end{array}\right]+k_{j}^{T}\left[\begin{array}{c}
n_{h_{1}}(l) \\
n_{h_{2}}(l) \\
\vdots \\
n_{h_{m}}(l)
\end{array}\right]
$$

where also the presence of the measurement noise has been considered. The expected value of the approximation error is given by

$$
E\left[\tilde{f}_{j}(l)-f_{j}(l)\right]=k_{j}^{T}\left[\begin{array}{c}
f_{h_{1}}(l) \\
f_{h_{2}}(l) \\
\vdots \\
f_{h_{m}}(l)
\end{array}\right]-f_{j}(l)
$$

while its standard deviation is given by

$$
\sigma\left[\tilde{f}_{j}(l)-f_{j}(l)\right] \leq \sqrt{k_{j}^{T} W^{T} W k_{j}}
$$

where

$$
W=\left[\begin{array}{cccc}
\sigma_{h_{1}}^{\prime \prime} & 0 & \cdots & 0 \\
0 & \sigma_{h_{2}}^{\prime \prime} & \cdots & 0 \\
\vdots & \vdots & \ddots & \vdots \\
0 & 0 & \cdots & \sigma_{h_{m}}^{\prime \prime}
\end{array}\right]
$$

For a better formalization of Problem 1, let us introduce the following definition.

Definition 1 . The $j$-th sensor is reconstructable from the sensors $h_{1}, h_{2}, \ldots, h_{m}$, if and only if the quadratic programming problem

$$
\begin{aligned}
& \min _{k_{j} \in \mathbb{R}^{\mathrm{m}}} k_{j}^{T} W^{T} W k_{j} \\
& \text { subject to } \\
& \left|k_{j}^{T}\left[\begin{array}{c}
f_{h_{1}}(l) \\
f_{h_{2}}(l) \\
\vdots \\
f_{h_{m}}(l)
\end{array}\right]-f_{j}(l)\right|<\sigma_{j}^{\prime}, \quad l=1,2, \ldots, N
\end{aligned}
$$


has a solution satisfying the inequality

$$
\sqrt{k_{j}^{T} W^{T} W k_{j}}<\sigma_{j}^{\prime}
$$

In other words, a measurement is reconstructable from the other measurements if:

- it can be approximated by means of a linear combination; the expected value of the approximation error is bounded by the minimum value of the standard deviation of the noise on the original signal;

- the standard deviation of the noise on the reconstructed measure is less or equal to the standard deviation of the noise affecting the original signal.

Exploiting Definition 1, Problem 1 can be tackled solving the following problem.

Problem 2. Find two partitions $P_{1}$ and $P_{2}$ of the set $\{1,2, \ldots, M\}$, such that the cardinality of $P_{2}$ is minimal and each measurement whose index is in $P_{1}$ is reconstructable from the measurements whose indices are in $P_{2}$.

Although the fact that, given two partitions $P_{1}$ and $P_{2}$, reconstructability of the measurement in $P_{1}$ from those in $P_{2}$ can be checked by solving a simple constrained quadratic programming problem, it is easy to show that Problem 2 is NP-hard. For this reason a suboptimal solution has been found following a heuristic approach, which led us to determine a set $P_{2}$ of cardinality 37 . In our optimal solution all the 30 flux sensors have been retained in the set $P_{2}$, while only 7 magnetic field sensors have been retained. The reason for this is that the magnetic field sensors are less accurate, and hence they are more easily reconstructable.

\section{Validation of the results with a plasma reconstruction code}

To validate the results obtained in the previous section, we used the EFIT++ code to reconstruct the plasma equilibrium for 50 configurations selected from equilibria database. EFIT++ (see [1],[4]) is a 2-D free boundary magnetic equilibrium reconstruction code, which can be configured to use a large set of diagnostic constraints. In this case it has been configured to use the ITER magnetic diagnostics in two kinds of simulations:

1. In the first type of simulation the plasma equilibrium has been reconstructed by using as EFIT++ inputs the actual values of the diagnostics corrupted by the assumed noise.

2. In the second type of simulation the plasma equilibrium has been reconstructed by using as EFIT++ inputs the actual values of the diagnostics corrupted by the assumed noise for the measurements in the partition $P_{2}$, and the reconstructed values for the measurement in the partition $P_{1}$.

As performance index we used the estimation errors on the six plasma-wall gaps shown in Fig. 2 (which are the gaps usually controlled in feedback by the ITER plasma shape controller). Fig. 3 shows the obtained results. In particular the increase in the estimation errors when the reconstructed measurements are used is negligible, confirming that it is possible to estimate these parameters by using a reduced set of measurements. 


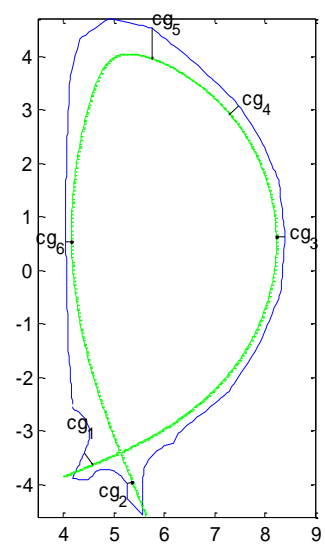

Fig. 2. The six ITER controlled gaps.
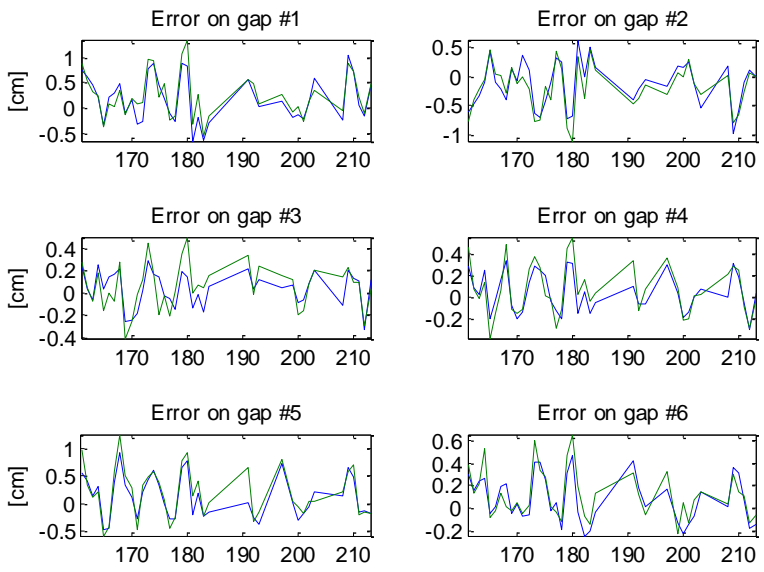

Fig. 3. Estimation error on the six controlled gaps using (a) the 78 actual measurements (blue), (b) the 37 actual measurements in $P_{2}$ and the 41 reconstructed measurements in $P_{1}$ (green).

\section{Conclusions}

In this paper we have presented a methodology to assess the minimum number of magnetic measurements which are necessary to reconstruct a plasma equilibrium for the ITER tokamak. Starting from the 78 primary magnetic sensors, we have shown that the use of 37 measurements leads to satisfactory results in terms of gap reconstruction, even in the presence of noise.

\section{References}

[1] L. C. Appel, et al., A unified approach to Equilibrium Reconstruction, in 33rd EPS Conference on Plasma Physics, Rome 2006.

[2] M. Ariola, A. Pironti, Magnetic Control of Tokamak Plasma, Springer Verlag London 2008

[3] A. Cenedese, P. Bettini, Assessment of the diagnostics for shape control in fusion machines, in Proc. of the 51st IEEE Conference on Decision and Control 2012

[4] L. L. Lao, et al., Reconstruction of current profile parameters and plasma shapes in tokamaks, Nuclear Fusion 25 (1985) 1611

[5] M. Shimada, et. al, Chapter 1: Overview and summary, Nuclear Fusion 47 (2007) S1 